\title{
Sexual orientation moderates the association between parental overprotection and stress biomarker profiles
}

\author{
Stephanie H. Cook (1) ${ }^{a}$, Jens C. Pruessner ${ }^{b, c}$, Sonia J. Lupien ${ }^{d}$ and Robert-Paul Juster \\ ${ }^{a}$ College of Global Public Health, New York University, New York, NY, USA; ${ }^{b}$ Department of Psychiatry, McGill \\ University, Montreal, Quebec, Canada; 'Department of Psychology, University of Constance, Constance, Germany; \\ 'Department of Psychiatry, University of Montreal, Montreal, Quebec, Canada; 'Department of Psychiatry, \\ Columbia University, New York, NY, USA
}

\begin{abstract}
Early experiences with parents may be particularly difficult for lesbian, gay and bisexual (LGB) individuals who face stigma that is linked to potentially distinct stress-related biobehavioural profiles. This study examined the association between parental bonding in relation to acute stress (cortisol reactivity) and chronic stress (allostatic load) in LGB and heterosexual individuals. The sample consisted of 87 healthy adults (mean [SD] age $=24.6$ [0.6] years; LGB: $n=46,43 \%$ women; and heterosexual $n=41,49 \%$ women). Regressions tested the main effects of retrospectively assessed parental overprotection and care before the age of 16 on stress reactive cortisol (area under the curve) and allostatic load (indexed using 21 neuroendocrine, immune, metabolic and cardiovascular biomarkers), and whether sexual orientation status moderated this association. Results revealed that parental overprotection was associated with increased cortisol reactivity only for LGB participants, but not for heterosexual participants. By contrast, parental overprotection was associated with higher allostatic load only for heterosexual participants, but not for LGB participants. While the functional significance of this requires further study, these preliminary findings suggest that adaptive processes among LGB individuals may mitigate the negative effects of parental overprotection on markers of chronic stress.
\end{abstract}

\section{KEYWORDS}

Parental bonding; sexual orientation; allostatic load; cortisol reactivity; sexual minority stress

\section{Introduction}

Our early relationships with a parent or primary caregiver are critical for the development of health and wellness throughout adulthood (Enns, Cox, \& Clara, 2002). Parental bonding describes the quality of this attachment that involves parenting styles that contribute to care (e.g. the extent to which a child feels warmth and nurtured by parents) and protection (e.g. the extent to which a child feels safe and protected by parents). By contrast, poor parental bonding is associated with greater psychological distress in adulthood (Lima, Mello, \& De Jesus Mari, 2010; Parker, 1988). For example, parental overprotection [e.g. the excessive maternal and/or paternal protection from the perspective of the child (Parker, 1983)] may convey to the child a sense that the world is dangerous, reinforce avoidance and limit their opportunities to develop skills and confidence in the face of life challenges (Clarke, Cooper, \& Creswell, 2013). Childhood parental overprotection is indeed associated with a myriad of negative health outcomes, including functional somatic symptoms in adolescence (Janssens, Oldehinkel, \& Rosmalen, 2009) and depression in adulthood (Irons, 
Gilbert, Baldwin, Baccus, \& Palmer, 2006). Parental overprotection is thought to negatively impact a child's ability to handle future social stressors in adulthood because it limits their opportunity to develop adaptive coping strategies (Janssens et al., 2009).

Research among lesbian, gay and bisexual (LGB) individuals suggests that they are more likely to experience poor parental relationships throughout adolescence than heterosexual individuals (Ryan, Russell, Huebner, Diaz, \& Sanchez, 2010). This may be attributed to complex interpersonal factors unique to LGB individuals, such as acceptance, or lack thereof, of their sexual orientation by parents that may disrupt the parental bonding process. For instance, Carnelley, Hepper, Hicks and Turner (2011) found that children of parents who were more accepting of their child's sexual orientation were more likely to have better romantic relationships in adulthood compared to children of parents who were less accepting. Further, D'Augelli, Grossman, Starks and Sinclair (2010) found that LGB adolescents with parents aware of their LGB status were less likely to report internalized homophobia compared to adolescents of unaware parents. Thus, the negative effects of stigma appear to strain those LGB youths who have not disclosed their orientation more so than those who have (Carragher \& Rivers, 2002).

Strained parental bonding among LGB youth may also be a risk factor for mental illness in adulthood. For instance, Rothman, Sullivan, Keyes and Boehmer (2012) found that poor parental relationships throughout childhood were associated with greater depression among gay and lesbian adults. Moreover, poor parental bonding may exacerbate the negative consequences of living in a less accepting context that can impede mental health. For example, Feinstein, Wadsworth, Davila and Goldfried (2014) found that fears of rejection and internalised homophobia were greater among LGB adults who had poorer parental relationships in childhood compared to LGB adults who had better childhood parental relationships. The extent to which parental bonding processes influence future patterns of psychosocial adjustment in adulthood among LGB individuals is of theoretical interest but has received limited empirical study.

\section{Integrated attachment and sexual minority stress model}

Attachment theory is an important framework for understanding how the formation of socioemotional bonds with parents and caregivers during childhood contributes to long-term development and functioning in adulthood. According to attachment theory, children develop relationships early on with parents or caregivers that shape the ways in which the child interacts with others and perceives the world (Ainsworth, 1989; Bowlby, 1969). Attachment researchers generally describe three attachment styles during childhood: anxious, avoidant and secure attachment. Children who are more secure in their attachment style tend to have healthy security attainment and proximity seeking behaviours (Ainsworth, 1989). On the other hand, children who are avoidant in their attachment style seem to be reluctant to be close to their caregivers during times of stress while those who are anxious in their attachment style experience great distress when separated from their caregiver and are difficult to comfort upon returning (Ainsworth, 1989). Children who have avoidant or anxious attachment orientations are considered to have an insecure attachment style.

In adulthood, research demonstrates that attachment can be characterised along the dimensions of anxiety and avoidance (Hazan \& Shaver, 1994). Adults who are low on both indices tend to have a more secure attachment style, while those who are higher on those indices tend to be more insecure. Adults who are more insecurely attached generally have interpersonal difficulties forming and maintaining relationships with others (Mikulincer \& Shaver, 2007). Studies of adult attachment generally find that adults that are more insecure in their attachment style and have higher levels of biological stress (e.g. cortisol) than adults that are more securely attached (Jaremka et al., 2013; Pietromonaco, DeBuse, \& Powers, 2013).

The sexual minority stress model (Meyer, 2003) provides a critical lens for understanding differences in the stress process between sexual minority and heterosexual individuals. The core 
assumption of this model is that the negative social evaluation of same-sex sexuality causes chronic stress in persons with a same-sex sexual orientation beyond the level of stress that people experience in general. This stress, in turn, causes unique and chronic stress that contributes to psychological and physical problems among LGB individuals. We have recently merged the sexual minority framework with an integrated attachment framework in order to understand LGB developmental trajectories so as to further our understanding of parental bonding processes.

The Integrated Attachment and Sexual Minority Stress or IASMS Model (Cook \& Calebs, 2016) provides a theoretical framework for understanding why there may be a greater magnitude in the association between poor parental bonding and minority stress experienced by LGB individuals. This theory posits that attachment relationships with parents, peers and romantic partners are shifted due to the influence of social stigma and discrimination that can be expressed by attachment figures. Therefore, as LGB individuals go through their sexual orientation identity formation and disclosure process, the expression of their non-heterosexual orientation can lead to rejection by parents and other attachment figures during young adulthood. This rejection and subsequent change in attachment can lead to increased perceived and physiological stress, which in turn is associated with poorer health in adulthood.

The IASMS model posits that sexual minority individuals may experience poorer parental bonding as a result of greater social stigma and discrimination based on their sexual orientation than non-sexual minority individuals. The IASMS model also proposes that this is associated with greater physiological stress. We believe that this is because sexual minority youths experience, in general, higher rates of attachment insecurity and parental rejection due to their sexual minority status which can lead to poor health and health behaviours in adulthood (Cook, Watkins, Calebs, \& Wilson, 2016). For instance, Rosario et al. (2014) found that attachment insecurity and maternal affection explained the association between sexual orientation and substance use. Further, they found that attachment insecurity mediated the association between sexual minority status and depression in their sample of sexual minority adults. In general, LGB youth are also more likely to have poor relationships with parents that contribute to poor mental health in adulthood compared to heterosexual youth (Pachankis, Goldfried, \& Ramrattan, 2008).

In summary, emerging research supports a link between poor parental relationships among LGB youth and poor mental health in adulthood. To date, however, it is less clear how parental bonding in childhood and early adolescence may influence disparities in stress physiology between LGB and heterosexual individuals in adulthood.

\section{Hypothalamic-pituitary-adrenal axis}

Sexual minority stress is believed to be associated with greater mental and physical health problems among LGB individuals compared to heterosexual individuals (Lick, Durso, \& Johnson, 2013). Researchers have begun to examine the association between sexual minority stress specifically in relation to physiological stress (Cook, Juster, Calebs, Heinze, \& Miller, 2017; Hatzenbuehler, 2009). Upon perceiving real or interpreted threats to homeostasis, the hypothalamic-pituitaryadrenal (HPA) axis produces cortisol that mobilises energy necessary for coping behaviours (Sapolsky, 2002). Dysregulation of the HPA axis is associated with psychological and physical health (Dedovic, Wadiwalla, Engert, \& Pruessner, 2009; Tsigos \& Chrousos, 2002). To assess these disease susceptibilities, laboratory stress paradigms (e.g. Trier Social Stress Test [TSST]) are used to understand individual differences in physiological responsivity to acute stress (Kirschbaum, Klauer, Filipp, \& Hellhammer, 1995; Kirschbaum, Wüst, \& Hellhammer, 1992).

HPA-axis reactivity to stressors is shaped by early experiences that have recently been explored among LGB individuals. A pioneering study by Hatzenbuehler and McLaughlin (2014) showed that LGB young adults that grew up in an area with high sexual orientation stigma and discrimination manifested blunted cortisol response in comparison to LGB youth who lived in more tolerant states. In addition to exaggerated HPA-axis reactivity, a blunted cortisol response may be indicative 
of trauma and fatigue (Fries, Hesse, Hellhammer, \& Hellhammer, 2005). Juster and colleagues (2015) showed a similar blunted cortisol reactivity among gay/bisexual men compared to heterosexual men in a Montreal sample. Interestingly, however, response patterns were inversed among lesbian/ bisexual women who showed greater post-stressor cortisol concentrations compared to heterosexual women (Juster et al., 2015). Because individual differences in stress reactivity studies are vast, a greater understanding of developmental factors (e.g. parental bonding) is required.

In studies conducted without regard for sexual orientation, early experiences with primary caregivers have been linked to long-term effects on health and well-being (Engert et al., 2010). Of particular interest is how childhood relationships with parents influence HPA-axis activation patterns (M. Pruessner, Vracotas, Joober, Pruessner, \& Malla, 2013). Initial research findings suggest that poor parental care is associated with heightened HPA-axis activation (Engert et al., 2010; Pruessner, Champagne, Meaney, \& Dagher, 2004). By contrast, a study conducted with a clinical population found that poor parental bonding was associated with a blunted cortisol response (Pruessner et al., 2013). To date, it remains unclear how the social and interpersonal features of one's life experiences influence inter-individual differences in HPA-axis reactivity among LGB individuals. The current study aims to provide further insights by linking parental bonding and sexual orientation to HPA-axis reactivity as well as cumulative physiological strain.

\section{Allostatic load}

The HPA axis mobilises energy necessary for adaptation, but at the cost of recalibrating many biological functions in turn (McEwen \& Wingfield, 2003). Compensatory alterations during acute stress include decreased digestion and bodily growth/repair counterbalanced to accommodate increased neurological, cardiovascular, respiratory and immune activities that are metabolically taxing. Over time, this can lead to widespread strain that dysregulates multiple interconnected systems in a pathophysiological process referred to as allostatic load.

Allostatic load is defined as the multi-systemic 'wear and tear' the brain and body endure under conditions of chronic stress (McEwen and Stellar, 1993). Allostatic load is measured by indexing biomarkers like cortisol as well as other neuroendocrine (e.g. dehyroepiandrosterone), immune (e.g. cytokines), metabolic (e.g. insulin) and cardiovascular (e.g. blood pressure) biomarkers related to stress physiology (Seeman, Singer, Rowe, Horwitz, \& McEwen, 1997). In studies throughout the world, allostatic load has been shown to predict physical and mental health outcomes better than individual biomarkers because of the subclinical and multi-systemic algorithms employed (Beckie, 2012; Juster, McEwen, \& Lupien, 2010). In the context of the current study, allostatic load has consistently been shown to be higher among socially disadvantages minority groups (Juster et al., 2011; Juster, Seeman et al., 2016).

To date, there are two studies that have assessed allostatic load as a function of sexual orientation. In a first Canadian study, gay/bisexual men surprisingly showed lower allostatic load than heterosexual men (Juster, Smith, Ouellet, Sindi, \& Lupien, 2013). In addition, LGB individuals who engaged less in avoidance coping strategies during their years of self-acceptance and disclosure of their sexual orientation to family and friends showed lower allostatic load (Juster, Ouellet et al., 2016). Indeed, those who had fully disclosed showed lower morning cortisol concentration and lower psychiatric symptoms than those who had not completed their 'coming out' process, often to family members (Juster et al., 2013). This finding suggests that the life experiences of sexual minorities may be the key to understanding their allostatic load trajectories. In accordance, stressful life events during childhood, adolescence and emerging adulthood were shown in a second study to further place LGB individuals at higher risk of allostatic load in an analysis of the American National Longitudinal Study for Adolescent Health (Hatzenbuehler, Slopen, \& McLaughlin, 2014).

In summary, allostatic load differs according to sexual orientation, presumably due to sexual minority stress processes that were not measured in either study. To the best of our knowledge, 
the extent to which parental bonding relates to allostatic load in either LGB or heterosexual individuals has not been investigated. The current study therefore aims to examine whether LGB individuals experience a greater magnitude of the association between poor parental bonding and $\mathrm{AL}$ than heterosexual individuals.

\section{Research objectives and hypotheses}

First, we aimed to understand the association of parental bonding on cortisol reactivity in response to a psychosocial stressor and allostatic load indexed using 21 stress-related biomarkers. Based on empirical literature linking childhood adversity to poorer mental health among LGB adults (IOM, 2011), we hypothesised that individuals who experienced parental overprotection would have distinct patterns of physiological stress reactivity and chronic stress in adulthood. Given that both hyper- and hypo-functioning of the HPA axis are deleterious to health (Fries et al., 2005), the directionality for stress reactivity was not explicitly hypothesised. Second, we aimed to understand if the association between poor parental bonding and physiological stress reactivity, as well as the association between poor parental bonding and chronic stress, would differ between LGB and heterosexual participants. Based on the IASMS model, we hypothesised that the association between poor parental bonding and dysregulated physiological stress indices and parental overprotection would be stronger for LGB participants than heterosexual participants.

\section{Methods}

\section{Participants}

Eighty-seven participants aged 18-45 years (mean $M$ [SE]; 24.61 [0.61]) identifying as lesbian/gay (8 women and 20 men), bisexual ( 12 women and 6 men) and heterosexual (20 women and 21 men) were recruited from the greater Montreal region as part of a larger study (Juster, Almeida et al., 2016; Juster, Ouellet et al., 2016; Juster et al., 2013; Juster et al., 2015). We used Internet advertisements, posters, liaisons among university community groups and word of mouth to generate a total pool of 257 prospects, of which 87 participated. The main exclusionary criteria were use of medicines affecting biomarkers (e.g. cortisone, anti-hypertensives), major health problems (e.g. cardiovascular disease, cancer, HIV/AIDS) and severe mental illness (e.g. schizophrenia, bipolar disorder).

To equalise groups because of disproportionately fewer lesbians and bisexual men, we combined sexual minorities by sex ( 20 women and 26 men) and contrasted them to heterosexuals ( 20 women and 21 men). Table 1 stratifies sample descriptive information according to demographics, socioeconomics, general health, well-being, lifestyle behaviours and interpersonal features of our sample as a function of sexual orientation and sex.

\section{Procedures}

This study was approved by the research ethics board of the Institut universitaire en santé mentale de Montréal on 28 April 2010 for a project entitled 'Étude psychoneuroendocrinienne de poids allostatique en milieu de travail'. Upon a 15-min study explanation and screening interview via telephone, eligible participants were scheduled for a first visit at the Centre for Studies on Human Stress. Visit 1 was scheduled between 8:00 AM and 11:00 AM and lasted approximately $40 \mathrm{~min}$. This visit involved written consent, blood draw from a certified nurse, a Continental breakfast to break a 12-h fast, completion of questionnaires (measuring parental bonding, sexual orientation, chronic stress and depressive symptoms) and, finally, detailed oral and written instructions for take-home urinary and salivary collection protocols. Participants were given take-home materials to collect saliva at five time-points throughout two non-consecutive days. Participants also completed the 
Table 1. Descriptive statistics for study sample.

\begin{tabular}{|c|c|c|c|c|}
\hline \multirow[b]{2}{*}{ Variable } & Heterosexual $(n=46)$ & $\operatorname{LGB}(n=41)$ & Total $(N=87)$ & \multirow[b]{2}{*}{$t / x^{2}$} \\
\hline & & $M(\mathrm{SD}) / N(\%)$ & & \\
\hline Age & $23.9(0.80)$ & $25.4(0.92)$ & $24.6(0.61)$ & -1.22 \\
\hline Sex & & & & 0.25 \\
\hline Men & $26(56.5 \%)$ & $21(51.2 \%)$ & $47(54.0 \%)$ & \\
\hline Women & $20(43.5 \%)$ & $20(48.8 \%)$ & $40(46.0 \%)$ & \\
\hline Race/Ethnicity & & & & 2.33 \\
\hline White/European & $36(78.3 \%)$ & $26(63.4 \%)$ & $62(71.3 \%)$ & \\
\hline Racial/Ethnic minority & $10(21.7 \%)$ & $15(36.6 \%)$ & $25(28.7 \%)$ & \\
\hline Education & & & & 6.67 \\
\hline High school & $40(87.0 \%)$ & 35 (87.5\%) & $75(87.2 \%)$ & \\
\hline No high school & $6(13.0 \%)$ & $5(12.5 \%)$ & $11(12.8 \%)$ & \\
\hline Income & & & & 1.11 \\
\hline$\leq 39,999$ USD & $34(73.9 \%)$ & $26(63.4 \%)$ & $60(69.0 \%)$ & \\
\hline$\geq 40,000$ USD & $12(26.1 \%)$ & $15(36.6 \%)$ & $27(31.0 \%)$ & \\
\hline Disclosure status & & & & $86.00^{* * *}$ \\
\hline Controls & $46(\% 100)$ & 0 & $41(\% 100)$ & \\
\hline Non-disclosed & 0 & $14(45.2 \%)$ & $14(45.2 \%)$ & \\
\hline Disclosed & 0 & $31(54.8 \%)$ & $31(54.8 \%)$ & \\
\hline Depressive symptoms & $8.61(5.00)$ & $8.63(9.32)$ & $8.62(7.31)$ & -0.02 \\
\hline Parental overprotection & $5.99(0.93)$ & $6.00(0.90)$ & $6.00(0.91)$ & -0.07 \\
\hline Parental care & $4.20(1.03)$ & $4.04(1.05)$ & $4.12(1.04)$ & 0.69 \\
\hline Cortisol reactivity $^{\mathrm{a}}$ & $-2.92(7.21)$ & $-1.48(6.06)$ & $-2.25(6.70)$ & -0.99 \\
\hline Allostatic load & $2.42(1.57)$ & $2.73(1.87)$ & $2.56(1.71)$ & -0.81 \\
\hline
\end{tabular}

questionnaires. Finally, participants were instructed to collect all urine produced over a 12-h period starting the night before returning to the laboratory.

Visit 2 was scheduled between 12:00 PM and 7:00 PM and lasted $120 \mathrm{~min}$. This visit included the return of completed saliva samples and the urine container subsequently frozen. A researcher took measurements of the waist and hip, as well as three seated resting measures of blood pressure for each participant. Visit 2 served mainly for exposure to the TSST, but participants also completed more questionnaires. Participants were then explained their blood results in detail and compensated with 50 Canadian dollars upon debriefing.

\section{Measures}

\section{Sexual orientation}

Sexual orientation was ascertained using three combined methods: (1) response to one of three separate advertisements searching for either lesbian/gay, bisexual or heterosexual participants; (2) asking participants their identified sexual orientation in an open-ended manner during telephone screening; and (3) administration of a modified 5-item Klein Sexual Orientation Scale (Klein, Sepekoff, \& Wolf, 1990). This instrument uses a 7-point Likert scale from 1 (other sex only) to 7 (same sex only) to assess sexual attractions, behaviours, sexual fantasies, preferences and identity. The entire sample's responses were internally consistent $(\alpha=0.982)$. Participants who identified as LGB were included in one category, while individuals who identified as heterosexual were included in a second category. The resultant variable used in our analyses was 'sexual orientation' $(0=L G B$, $1=$ heterosexual).

\section{Parental bonding}

The Parental Bonding Inventory (Parker, 1988) is a 50-item self-report measure that evaluates perceptions concerning parental care and overprotection before the age of 16. Questions were asked separately for mothers and fathers. The overprotection subscale comprised 24 items and was rated on a 7-point Likert scale, with lower scores indicating less overprotection and greater 
scores indicating more overprotection. The care subscale comprised 26 -items and was also rated on a 7-point Likert scale. Mother and father measures were averaged for both the overprotection and care subscales. High scores indicated more parental care, while lower scores indicated less care. We combined the measures of mother and father, and thus, the resultant measure evaluated overall parental overprotection and overall parental care. The measure of parental bonding, including total overprotection and total care, showed suitable internal reliability, $a$ $=0.87$ and 0.91 , respectively.

\section{Cortisol reactivity}

Exposure to a modified version (Andrews et al., 2007; Wadiwalla et al., 2010) of the TSST (Kirschbaum, Pirke, \& Hellhammer, 1993) occurred during a 2-h afternoon visit to our laboratory. After a 10-min anticipation phase, participants were led to a separate room where they were asked to perform a 5-min mock job interview followed by $5 \mathrm{~min}$ of mental arithmetic in front of an unseen, ostensible behavioural expert seated behind a one-way mirror. Further details for validation of this permutation have been previously reported (Andrews et al., 2007; Marin et al., 2012; Wadiwalla et al., 2010). The participant and the 'behavioural expert' communicated via an intercommunication device, and the participant's performance was recorded by a video camera. A seminal meta-analysis (Dickerson \& Kemeny, 2002) posits that laboratory-based stressors eliciting social-evaluative threat include evaluative audiences, negative social comparisons or recorded performance that maximise HPA-axis reactivity.

To determine cortisol concentrations before and after TSST exposure, 10 saliva samples were collected at 10-min intervals via the passive drool method with Salivettes ${ }^{\circledR}$. All assays were conducted at the Centre for Studies on Human Stress (www.humanstress.ca) in Montreal, Quebec, Canada. Prior to assaying each biomarker, frozen samples were brought to room temperature and then centrifuged at $1500 \times g(3000 \mathrm{rpm})$ for $15 \mathrm{~min}$. For cortisol determination, we used a high-sensitivity enzyme immune assay kit (Salimetrics ${ }^{\circledR}$ State College, PA, Catalogue No. 1-3102), with the range of detection between 0.012 and $3 \mu \mathrm{g} / \mathrm{dL}$. Inter-assay and intra-assay coefficients of variance were, respectively, below $10.08 \%$ and $8.58 \%$ for cortisol. Duplicate assays and raw data were averaged.

Time-dependent stress reactive cortisol was calculated using area under the curve with respect to increase (AUCi) formulae derived from the trapezoid formula (Pruessner, Kirschbaum, Meinlschmid, \& Hellhammer, 2003). The AUCi captures increases from baseline in cortisol concentrations throughout the TSST.

\section{Allostatic load}

Twenty-one biomarkers representing neuroendocrine, immune, metabolic and cardiovascular functioning were incorporated into an allostatic load index (Juster et al., 2015; Juster, Ouellet et al., 2016).

For neuroendocrine biomarkers, we included salivary diurnal cortisol sampled on two nonconsecutive workdays at five time-points (awakening, +30 min after awakening, 14:00, 16:00 and at bedtime) that was transformed into both AM cortisol and PM cortisol slopes to capture dynamic diurnal HPA-axis fluctuations. We also included serum dehydroepiandrosterone sulphate, and 12-h overnight urinary adrenalin, noradrenalin and dopamine.

For immune/inflammatory biomarkers, we included plasma interleukin- 6 and tumour necrosis factor-alpha, serum c-reactive protein and serum fibrinogen preserved with sodium citrate.

For metabolic biomarkers, we included serum albumin, creatinine, insulin, glycosylated haemoglobin (in per cent), total cholesterol, high-density lipoprotein and triglycerides, as well as waist-tohip ratio measured with graduated tape and calculated by dividing respective inches and body mass index calculated as mass (in kilograms) divided by height (in metres squared).

For cardiovascular biomarkers, we used the mean of three measures of resting seated systolic and diastolic blood pressure recordings measured with an electronic sphygmomanometer (A\&D Medical: Model UA-631V). 
Participants' values were coded with respect to clinical reference ranges and aggregated into an allostatic load index (Juster et al., 2010, 2011). This clinically based or 'norm allostatic load index' formulation is derived from population-based cut-offs for biomarker distributions rather than sample-based cut-offs as originally used in validation of the allostatic load model (Seeman et al., 1997). Percentiles were calculated based on clinical reference ranges whereby values passing the highest 75th percentile were scored as ' 1 ', and those falling below the 75 th percentile were scored as ' 0 '. Two exceptions to this formulation were applied: (a) the lowest 25 th percentile denotes high risk for dehydroepiandrosterone sulphate, albumin and high-density lipoprotein cholesterol and (b) because hypocortisolism and hypercortisolism are both deleterious (Fries et al., 2005), two-tailed formulations set at 12.5 and 87.5 percentiles based on the sample's distribution were used for diurnal cortisol measures. In sum, the allostatic load index was operationally defined as the number of dysregulated biomarkers that could range from 0 to 21 .

\section{Covariates}

Covariates included sex, age, race/ethnicity, depressive symptoms and disclosure status as justified by existing literature. Biological sex drives variability in biobehavioural responsivity to stress (Taylor et al., 2000) and numerous human studies demonstrate that men show higher free cortisol concentrations in response to psychosocial stressors than women of reproductive age (Kajantie \& Phillips, 2006). Ageing is also a known predictor of increased allostatic load because of the physiological toll that chronic stress exerts on the brain and body over time (Crimmins, Johnston, Hayward, \& Seeman, 2003). Moreover, evidence suggests that there may be differences in stress and stress reactivity by race/ethnicity (Yanovski, Yanovski, Harrington, Gold, \& Chrousos, 1995). Thus, we controlled for potential racial/ethnic differences in the analyses.

HPA-axis activation is also associated with clinical and non-clinical psychiatric symptoms such as depression (Burke, Davis, Otte, \& Mohr, 2005). Thus, the 21-item Beck Depression Inventory II (BDI) (Beck, Steer, \& Brown, 1996) was administered to assess depressive symptoms. The BDI utilises a 4point Likert scale ranging from 0 to 3 in accordance to statements pertaining to depressive symptoms over the last 2 weeks. In the current study, a total sum BDI score revealed acceptable internal consistency for both sexual minorities $(a=0.76)$ and heterosexual individuals $(a=0.94)$.

We also designed a brief four-item questionnaire administered only to sexual minority participants ( $n=46)$ in order to measure disclosure status. Instructions were as follows: 'Please try to remember the feelings and circumstances you experienced through the process of identification and acceptance of your sexual orientation'. Participants responded to the following four questions: (A) 'How old were you when you first recognised same-sex attractions?', (B) 'How old were you when you conclusively identified your sexual orientation?', (C) 'At what age, if at all, did you "come out" to friends?' and (D) 'At what age, if at all, did you "come out" to family?' Disclosure status was coded as 'disclosed' if A, B, C and $D$ were all answered. Participants were coded as 'non-disclosed' if one or more questions were not answered, or if were refuted with statements such as 'only my cousins know and not my parents'. Disclosed $(n=31)$ and non-disclosed $(n=14)$ groups were assigned irrespective of one's biological sex, and heterosexual participants were included as controls $(n=41)$.

Lastly, we considered including contraceptive use in our model due to its association with cortisol among biological females (Kirschbaum, Kudielka, Gaab, Schommer, \& Hellhammer, 1999); however, we conducted an independent $t$-test and found that there was no difference between women who used contraceptives and women who did not on their $\mathrm{AUCi}, t(52)=1.48, p=0.15$. Therefore, we did not include contraceptive use in the final regression models.

\section{Statistical analysis}

We conducted descriptive statistics with the full sample and with the sample stratified by sexual orientation in order to understand the association among the chosen covariates, parental bonding 
and biological stress indices. Parental care and parental overprotection were both normally distributed. Moderation analyses were conducted using the PROCESS SPSS macro version 3 in SPSS Statistics Version 25 (Hayes, 2008). PROCESS is a computational tool that generates $95 \%$ biascorrected confidence intervals and conditional effect estimates [i.e. estimates under which condition(s) the moderation effect is significant (Hayes, 2008)]. Four moderation models were conducted using 5000 bootstrapped samples: two models examined the indirect effect of sexual orientation on the association between parental overprotection and care and cortisol reactivity, respectively. The other two models examined the indirect effect of sexual orientation on the association between parental overprotection and care on allostatic load, respectively. All analyses controlled for sex, race/ethnicity, age, depressive symptoms and disclosure status. Lastly, we conducted a post-hoc power analysis using G*Power version 5 (Faul, Erdfelder, Buchner, \& Lang, 2009) for a linear regression model based on study parameters.

\section{Results}

\section{Sample characteristics}

Table 1 provides information on the sample characteristics. The majority of participants identified racially/ethnically as White/European (78.3\%) and ranged in age from 18 to 45 years old $(M=24.6$, SD $=0.61)$. The sample was composed of about equal numbers of men and women. Eighty-seven per cent of heterosexual individuals and $87.5 \%$ of LGB individuals reported completing high school. There were no overall differences in parental bonding, cortisol reactivity or allostatic load between heterosexual and LGB participants. For descriptive purposes, Figure 1 illustrates cortisol reactivity and allostatic load by sexual orientation group while controlling for sex, race/ethnicity, depressive symptoms and disclosure status.

\section{Cortisol reactivity}

In Model 1, we examined if sexual orientation had an indirect effect on the association between parental overprotection and cortisol reactivity (Table 2). Model 1 was statistically significant $\left(F_{(9,71)}=2.31, p=0.02\right)$ and explained $23 \%$ of the variance in cortisol reactivity. In comparison to White/European participants, non-White participants were significantly more likely to have lower AUCi $(b=-3.43, p=0.04)$. Higher levels of depressive symptoms were associated with lower AUCi $(b=-0.29, p=0.01)$, and greater levels of parental overprotection were associated with higher AUCi $(b=3.08, p=0.01)$. Further, sexual orientation significantly
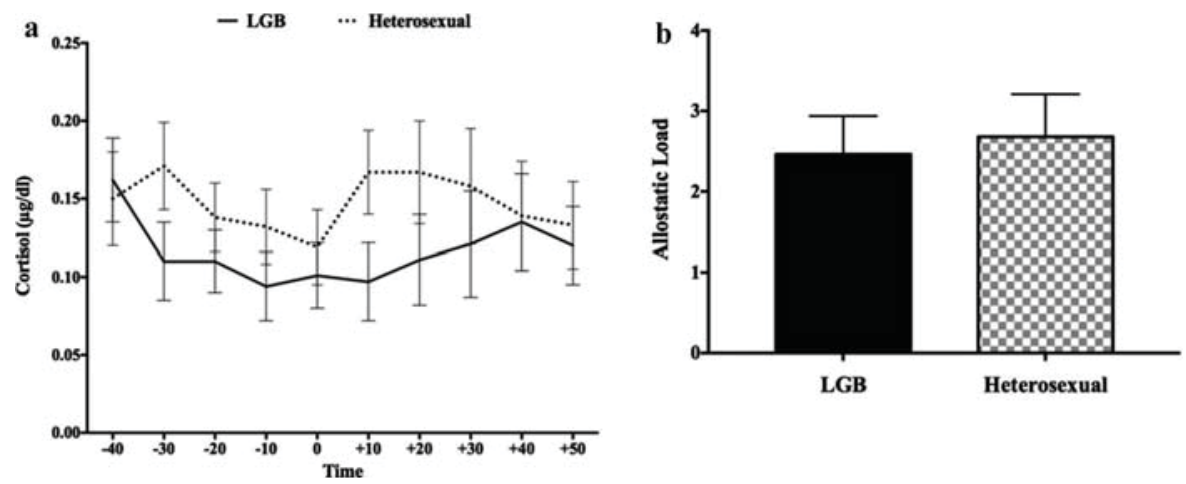

Figure 1. Salivary cortisol (a) and allostatic load (b) among lesbian, gay and bisexual (LGB) compared to heterosexual individuals. 
Table 2. Linear regression models examining the associations between parental bonding and stress indices.

\begin{tabular}{|c|c|c|c|c|c|c|}
\hline & \multirow{2}{*}{\multicolumn{2}{|c|}{$\begin{array}{c}\text { Cortisol reactivity (AUCi) } \\
\text { Model } 1\end{array}$}} & \multicolumn{4}{|c|}{ Allostatic load (AL) } \\
\hline & & & \multicolumn{2}{|c|}{ Model 2} & \multicolumn{2}{|c|}{ Model 3} \\
\hline & $b$ (SE) & $95 \% \mathrm{Cl}$ & $b(\mathrm{SE})$ & $95 \% \mathrm{Cl}$ & $b(\mathrm{SE})$ & $95 \% \mathrm{Cl}$ \\
\hline Age & $-0.11(0.13)$ & $-0.37,0.16$ & $0.10(0.03)^{* *}$ & $0.04,0.16^{* *}$ & $0.09(0.03)^{*}$ & $0.02,0.15$ \\
\hline $\operatorname{Sex}^{a}$ & $1.07(1.47)$ & $-1.86,4.00$ & $0.20(0.35)$ & $-0.49,0.89$ & $0.34(0.36)$ & $-0.37,1.06$ \\
\hline Depressive symptoms & $-0.29(0.11)^{* *}$ & $-0.50,-0.08^{* *}$ & $0.11(0.03)^{* * *}$ & $0.06,0.16^{* * *}$ & $0.10(0.03)^{* *}$ & $0.05,0.16$ \\
\hline Disclosure status ${ }^{b}$ & $-1.63(2.25)$ & $-6.11,2.85$ & $-0.10(0.53)$ & $-1.16,0.95$ & $-0.27(0.55)$ & $-1.37,0.83$ \\
\hline Race $^{c}$ & $-0.52(0.24)^{*}$ & $-1.00,-0.04^{*}$ & $0.50(0.38)$ & $-0.27,1.26$ & $0.45(0.40)$ & $-0.35,1.24$ \\
\hline PO & $3.08(1.13)^{*}$ & $0.83,5.33^{*}$ & $-0.17(0.27)$ & $-0.70,0.36$ & $0.30(0.20)$ & $-0.11,0.71$ \\
\hline PC & $0.59(0.77)$ & $-0.95,2.12$ & $0.11(0.18)$ & $-0.25,0.48$ & 0.26 & $-0.23,0.75$ \\
\hline $\mathrm{SO}^{\mathrm{d}}$ & $24.41(10.14)^{*}$ & $4.20,44.62^{*}$ & $-6.00(2.39)^{*}$ & $-10.76,-1.24^{*}$ & 0.75 & $-3.07,4.57$ \\
\hline $\mathrm{PO} \times \mathrm{SO}$ & $-4.06^{*}$ & $-7.36,-0.76^{*}$ & $1.00(0.39)^{*}$ & $0.22,1.77^{*}$ & - & - \\
\hline $\mathrm{PC} \times \mathrm{SO}$ & - & - & - & - & -0.25 & $-0.95,0.46$ \\
\hline \multicolumn{7}{|l|}{ Conditional effects } \\
\hline Straight & $-0.98(1.22)$ & $-3.42,1.45$ & $0.83(0.29)^{*}$ & $0.26,1.40^{*}$ & - & - \\
\hline LGB & $3.08(1.13)^{* *}$ & $0.83,5.33^{* *}$ & $-0.17(0.27)$ & $-0.70,0.36$ & - & - \\
\hline$R^{2}$ & 0.23 & & 0.37 & & 0.32 & \\
\hline
\end{tabular}

${ }^{* * *} p<0.0001 ;{ }^{* *} p<0.01 ;{ }^{*} p<0.05$. AUCi: Area under the curve with respect to increase; PO: parental overprotection; PC: parental care; SO: sexual orientation.

${ }^{\text {a }}$ The referent group is men.

${ }^{\mathrm{b}}$ The referent group is the disclosure (LGB individual only).

'The referent group is White.

${ }^{\mathrm{d}}$ The referent group is straight.

moderated the association between parental overprotection and cortisol reactivity $(b=-4.06$, $p=0.02$ ). Figure 2 reveals that parental overprotection was associated with higher cortisol reactivity among LGB participants $(m=3.08, p=0.01)$, but not among heterosexual participants ( $m=-0.98, p=0.42$ ).

The model examining if sexual orientation had an indirect effect on the association between parental care and cortisol reactivity was not statistically significant $\left(F_{(9,71)}=1.51, p=0.16\right)$.

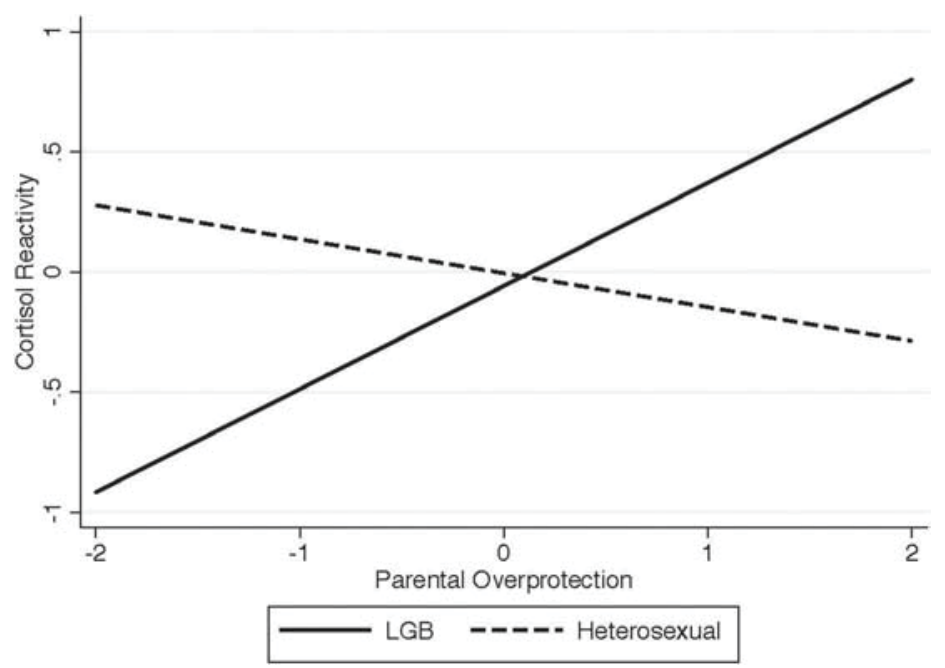

Figure 2. Conditional effects of parental overprotection on cortisol reactivity between LGB and heterosexual participants. 


\section{Allostatic load}

Model 2 examined if sexual orientation had an indirect effect on the association between parental overprotection and allostatic load (Table 2). Model 2 was statistically significant $\left(F_{(9,71)}=4.64\right.$, $p<0.0001)$ and explained $37 \%$ of the variance in allostatic load. Results indicated that those who were older $(b=1.01, p=0.002)$ and who had more depressive symptoms $(b=0.11, p<0.0001)$ both had significantly higher allostatic load. Further, the interaction between sexual orientation and parental overprotection was significant $(b=1.00, p=0.01)$. As displayed in Figure 3 , conditional effects revealed that greater parental overprotection was associated with higher allostatic load among heterosexual participants ( $m=0.83, p=0.005)$ but not among LGB participants ( $m=-0.17, p=0.53$ ).

Model 3 displays the results for the model examining the indirect effect of sexual orientation on the association between parental care and allostatic load. The model was significant $\left(F_{(9,71)}=2.67\right.$, $p=0.0008)$ and indicated that sexual orientation was not a significant moderator $(b=-0.25$, $p=0.49)$.

\section{Post-hoc power analysis}

A post-hoc power analysis was computed to determine an appropriate sample size for a linear regression model predicting mean IMT from the independent variables. A sample size of 136 achieves $80 \%$ power to detect an $R^{2}$ of 0.05 attributed to 1 independent variable using an $F$-test with $a=0.05$. The variables tested are adjusted for an additional eight independent variables with an $R^{2}$ of 0.10 . In our study, a sample size of 87 participants and effect size $F^{2}$ of 0.15 yielded power of $65 \%$.

\section{Discussion}

The current preliminary analysis shows that sexual orientation moderates the association between parental overprotection and stress biomarker profiles of acute stress (cortisol reactivity) and chronic stress (allostatic load). By contrast, there were no statistically significant findings for parental care. Overall, our main effects without regards for sexual orientation showed no association between parental overprotection during childhood and later stress reactivity or allostatic load.

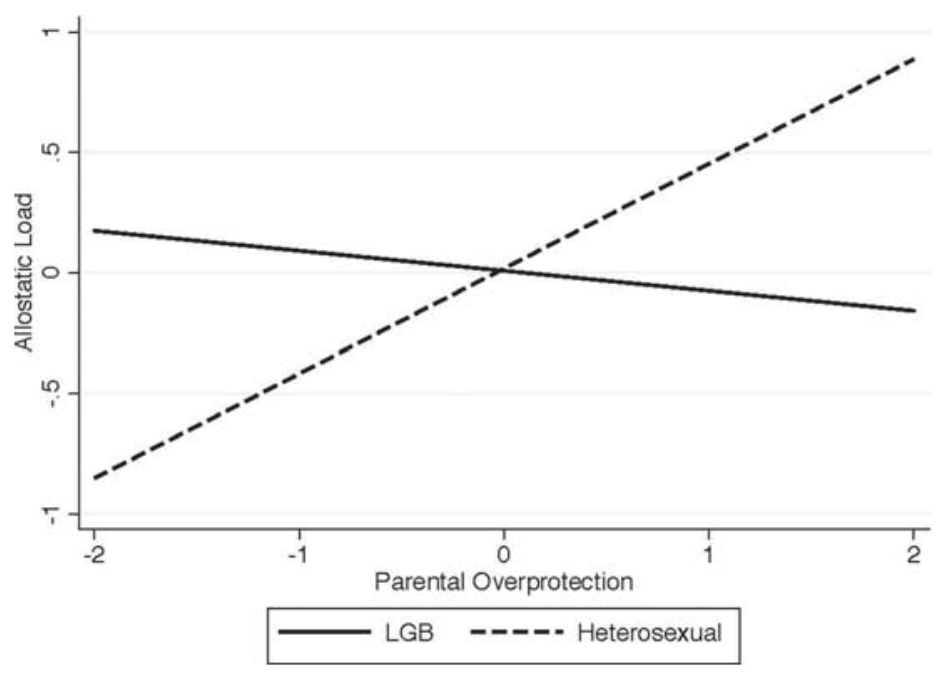

Figure 3. Conditional effects of parental overprotection on allostatic load between LGB and heterosexual participants. 
Due to sexual minority stress and the heightened rates of childhood familial adversity previously reported among LGB individuals (Corliss, Cochran, \& Mays, 2002; Katz-Wise \& Hyde, 2012; Pachankis et al., 2008; Saewyc, Skay, Pettingell, \& Reis, 2006), we hypothesised that LGB participants might demonstrate a higher effect magnitude between parental overprotection and cortisol reactivity either hyper- or hypo-active profiles - as well as allostatic load. This hypothesis was only partially supported in the context of acute stress for LGB individuals. Specifically, overprotective parental relationships were positively associated with stress reactivity for LGB adults in comparison to heterosexual individuals that did not show this association. Because this moderation effect among LGB individuals emerges in spite of no main effects and a minimal HPA-axis response, we must be cautious about conclusions. For chronic stress, there was no association between parental relationships and chronic stress among LGB participants; however, there was for heterosexuals.

While our results are preliminary and of low power, we speculate that the moderation results represent differential profiles of physiological stress processes among LGB and heterosexual individuals. This could in turn modulate the association between parental overprotection and distinct profiles of acute versus chronic physiological stress. In particular, our allostatic load findings suggest that heterosexual participants who had overprotective parents were more likely to experience chronic stress in adulthood, a finding that was not observed among the LGB participants. This is contrary to our hypothesis, which was guided by the theoretical assumption that sexual minority individuals experience stronger associations between parental overprotection and poor health as compared to heterosexual individuals due to their exposure to sexual orientation-related stigma and discrimination.

There are several potential explanations for our allostatic load findings, which relate to the concepts of desensitisation and resilience. First, it is plausible that over time, LGB youth become desensitised to the effects of sexual orientation-related social stress and discrimination due to recurrent exposure. Desensitisation may arise, in part, through the type of relationship that LGB youths form with parents/caregivers. LGB youth who have parents that are overprotective may experience desensitisation to stressors more strongly than those who do not have overprotective parents. In addition, LGB youth may simultaneously experience excess stress due to social stigma and discrimination throughout development which can cumulatively affect their sensitivity to social stressors.

Second, it may be the case that due to heightened levels of sexual minority stress, some LGB individuals become resilient and thus do not experience the negative effects of parental overprotection on chronic stress. Researchers have only recently begun to examine how resilience may contribute to positive health outcomes among LGB individuals despite experiencing sexual minority stress (Herrick, Stall, Goldhammer, Egan, \& Mayer, 2014). Consistent with sexual minority stress, these unique stressors can also motivate sexual minorities to seek social support and resources that can promote adaptive coping strategies and resilience for individuals and communities (Meyer, 2016). A study showed that LGB young adults who felt more familial social support experienced lower cortisol stress reactivity (Burton, Bonanno, and Hatzenbuehler, 2014). Further empirical research on how social support and resilience can promote positive health among LGB individuals requires longitudinal designs. Therefore, future research should focus on understanding how adaptive processes mitigate the negative effects of stigma independent of parental bonding.

Further, results from our study suggest that overprotective parenting is associated with stress reactivity, but not chronic stress, among LGB young adults. Future research should focus on examining the influence of sexual orientation discrimination as one possible factor influencing stress reactivity among LGB populations. To better understand nuances in parental bonding among LGB individuals compared to heterosexual individuals, future prospective research should also identify non-familial protective factors that promote positive health outcomes. Specifically, how does social support outside the family synergise or supersede attachment styles originally developed with parents? How might a family that is supportive of one's sexual minority identity correspond to distinct biological stress profiles? While the current analysis cannot address these 
questions, we can speculate that there are key processes during sexual identity formation that occur in conjunction with other sexual minorities undergoing similar experiences that can function as key sources of support among LGB individuals.

To the best of our knowledge, this is the first study to show differences between sexual minorities and heterosexual individuals in the parental bonding and stress physiology literature. Because LGB individuals experience high levels of stigma and discrimination (Hatzenbuehler, 2009), they may also have strained relationships with their families that may not be supportive of their minority sexual identity (Cook \& Calebs, 2016; Rosario et al., 2014). Past research has shown that childhood adversity is associated with increased stress reactivity (Hunter, Minnis, \& Wilson, 2011). However, these studies, and the current study, have not specifically examined how sexual orientation-related stigma and discrimination may differentially influence the association between parental relationships and physiological stress in adulthood. In addition, there are important subgroup differences among the LGB community and by sex that may correspond to unique parental bonding processes. Future studies should examine if stigma and discrimination differentially influences the association between early parental bonding and later physiological stress among LGB subgroups and heterosexual individuals.

The current study has several limitations. First, the sample consists mostly of White individuals, and thus, the findings cannot be generalised to individuals of different racial/ethnic backgrounds. There is also considerable heterogeneity in our sample that we could not adequately nuance as a function of sexual orientation subgroups and sex. While many studies have likewise combined groups according to sex to preserve statistical power (IOM, 2011), there are critical distinctions within the LGB community in health disparities that could influence cortisol reactivity and allostatic load. A study by Everett and colleagues (2014) examining inflammation and immune functioning and sexual orientation found evidence that sexual minority men may exhibit higher levels of biological stress than sexual minority women. However, other studies have found no significant differences in stress profiles among sexual minority males versus females (Austin et al., 2016). Thus, it is important that researchers further study these potential distinctions in stress physiology among LGB populations.

Second, because our study consists of a relatively small sample size that tested two moderation models, we may have increased the chance of Type I error. Because our post-hoc power analysis yield a result of $65 \%$, we can only consider these results to be preliminary that will need to be replicated with better powered studies. Third, the retrospective properties of the parental bonding measure may be considered a limitation of the measurement. However, the measure of parental bonding used has been validated in clinical and non-clinical populations (Parker, 1988). Fourth, we constructed our hypotheses utilising an integrated attachment and sexual minority framework (Cook \& Calebs, 2016); however, we did not explicitly measure LGB-related stigma. Several studies show that sexual orientation-related discrimination is a key driver of the health disparities observed between LGB and heterosexual individuals and thus warrants further exploration within the context of parental bonding and later physiological stress (Saewyc, 2011).

\section{Conclusions}

Limitations notwithstanding, our study is the first to examine differences between parental bonding and the physiological stress processes among heterosexual and LGB individuals. In summary, we found that the association between parental overprotection and biological stress indices is moderated by sexual orientation. This association is manifested among LGB individuals under conditions of acute stress. On the other hand, this association is manifested cumulatively among heterosexual individuals under conditions of chronic stress. Future research should prospectively assess both risk and protective factors involved.

Our preliminary results have potential implications for continued efforts to improve the health and well-being of LGB youth. While our study represents a first step, we suggest that future 
research focus on how to promote secure relationships between youth and parents in the context of diverse sexual orientations and gender identities. Further research must be conducted to critically examine the specific developmental and social mechanisms linking parental bonding and physiological stress among LGB. This will lead to a better understanding of exactly how to specify better health policies and interventions. As posited by the IASMS model, underlining social stigma and discrimination may directly impact the parental relationships of LGB youth and their parents in ways that can buffered against.

\section{Acknowledgements}

We thank our participants for their commitment to this demanding study. Many thanks go to Helen Findlay and Edouard Kouassi for performing biochemical assays. This project was possible with the nurse services of Louise Normandeau, Soaud Lahlafi and Carole Feltrin.

\section{Disclosure statement}

No potential conflict of interest was reported by the authors.

\section{Funding}

This study was funded by the Canadian Institutes of Health Research [Grant Number 222055] to S.J.L. who also held a Senior Investigator Chair on Gender and Mental Health from the Canadian Institute of Gender and Health (GSC 91039). R. P. Juster is currently the recipient of the Canadian Institutes of Health Research Banting Postdoctoral Fellowship.

\section{Notes on contributors}

Dr. Stephanie $H$. Cook studies the pathways and mechanisms linking attachment, minority stress, and health among disadvantaged individuals. She examines how the inter- and intra- personal features of close relationships influence the health of racial/ethnic and sexual minorities.

Dr. Jens C. Pruessner studies stress and aging, using various techniques ranging from the self-developed Montreal Imaging Stress Task, over the cortisol response to awakening, to protocols allowing the assessment of specific structures in the medial temporal lobes with help of Magnetic Resonance Imaging.

Dr. Sonia J. Lupien studies the effects of stress throughout life. Her studies have shown that children are vulnerable to the effects of stress, young adults under acute stress show impairments in memory and emotional regulation, and finally that chronic stress among older adults is associated with hippocampal atrophy, a region involved in learning and memory processes.

Dr. Robert-Paul Juster's research focuses on chronic stress among diverse social groups using transdisciplinary approaches that tease apart biological sex and socio-cultural gender. He has developed expertise in measuring chronic stress known as allostatic load that describes the physiological dysregulations related to chronic stress and unhealthy behaviors.

\section{ORCID}

Stephanie H. Cook (iD) http://orcid.org/0000-0003-4505-0813

\section{References}

Ainsworth, M. (1989). Attachments beyond infancy. American Psychologist, 44, 709-716.

Andrews, J., Wadiwalla, M., Juster, R. P., Lord, C., Lupien, S. J., \& Pruessner, J. C. (2007). Effects of manipulating the amount of social-evaluative threat on the cortisol stress response in young healthy men. Behavioral Neuroscience, 121(5), 871-876. 
Austin, S. B., Rosario, M., McLaughlin, K. A., Roberts, A. L., Gordon, A. R., Sarda, V., ... Scherer, E. A. (2016). Sexual orientation and diurnal cortisol patterns in a cohort of US young adults. Psychoneuroendocrinology, 69, 197208.

Beck, A. T., Steer, R. A., \& Brown, G. K. (1996). Manual for the beck depression inventory-ii. San Antonio, TX: Psychological Corporation.

Beckie, T. M. (2012). A systematic review of allostatic load, health, and health disparities. Biological Research For Nursing, 14(4), 311-346.

Bowlby, J. (1969). Attachment and loss: Vol. 1. Attachment. New York: Basic Books.

Burke, H. M., Davis, M. C., Otte, C., \& Mohr, D. C. (2005). Depression and cortisol responses to psychological stress: A meta-analysis. Psychoneuroendocrinology, 30(9), 846-856.

Carnelley, K. B., Hepper, E. G., Hicks, C., \& Turner, W. (2011). Perceived parental reactions to coming out, attachment, and romantic relationship views. Attachment \& Human Development, 13, 217-236.

Burton, C. L., Bonanno, G. A., \& Hatzenbuehler, M. L. (2014). Familial social support predicts a reduced cortisol response to stress in sexual minority young adults. Psychoneuroendocrinology, 47, 241-245. https://doi.org/10.1016/j.psy neuen.2014.05.013

Carragher, D. J., \& Rivers, I. (2002). Trying to hide: A cross-national study of growing up for non-identified gay and bisexual male youth. Clinical Child Psychology and Psychiatry, 7, 457-474.

Clarke, K., Cooper, P., \& Creswell, C. (2013). The parental overprotection scale: Associations with child and parental anxiety. Journal of Affective Disorders, 151(2), 618-624.

Cook, S. H., \& Calebs, B. J. (2016). The Integrated attachment and sexual minority stress model: Understanding the role of adult attachment in the health and well-being of sexual minority men. Behavioral Medicine, 42, $164-173$.

Cook, S. H., Juster, R.-P., Calebs, B. J., Heinze, J., \& Miller, A. L. (2017). Cortisol profiles differ by race/ethnicity among young sexual minority men. Psychoneuroendocrinology, 75, 1-4.

Cook, S. H., Watkins, D., Calebs, B. J., \& Wilson, P. A. (2016). Attachment orientation and sexual risk behaviour among young Black gay and bisexual men. Psychology \& Sexuality, 7, 177-196.

Corliss, H. L., Cochran, S. D., \& Mays, V. M. (2002). Reports of parental maltreatment during childhood in a United States population-based survey of homosexual, bisexual, and heterosexual adults. Child Abuse \& Neglect, 26, 11651178.

Crimmins, E. M., Johnston, M., Hayward, M., \& Seeman, T. E. (2003). Age differences in allostatic load: An index of physiological dysregulation. Experimental Gerontology, 38, 731-734.

D'Augelli, A. R., Grossman, A. H., Starks, M. T., \& Sinclair, K. O. (2010). Factors associated with parents' knowledge of gay, lesbian, and bisexual youths' sexual orientation. Journal of GLBT Family Studies, 6, 178-198.

Dedovic, K., Wadiwalla, M., Engert, V., \& Pruessner, J. C. (2009). The role of sex and gender socialization in stress reactivity. Developmental Psychology, 45, 45-55.

Dickerson, S. S., \& Kemeny, M. E. (2002). Acute stressors and cortisol reactivity: A meta-analytic review. Psychosomatic Medicine, 54, 105-123.

Engert, V., Buss, C., Khalili-Mahani, N., Wadiwalla, M., Dedovic, K., \& Pruessner, J. C. (2010). Investigating the association between early life parental care and stress responsivity in adulthood. Developmental Neuropsychology, 35, 570-581.

Enns, M. W., Cox, B. J., \& Clara, I. (2002). Parental bonding and adult psychopathology: Results from the US National Comorbidity Survey. Psychological Medicine, 32(06), 997-1008.

Everett, B. G., Rosario, M., McLaughlin, K. A., \& Austin, S. B. (2014). Sexual orientation and gender differences in markers of inflammation and immune functioning. Annals of Behavioral Medicine, 47, 57-70.

Faul, F., Erdfelder, E., Buchner, A., \& Lang, A.-G. (2009). Statistical power analyses using G*Power 3.1: Tests for correlation and regression analyses. Behavior Research Methods, 41(4), 1149-1160.

Feinstein, B. A., Wadsworth, L. P., Davila, J., \& Goldfried, M. R. (2014). Do parental acceptance and family support moderate associations between dimensions of minority stress and depressive symptoms among lesbians and gay men? Professional Psychology: Research and Practice, 45, 239-246.

Fries, E., Hesse, J., Hellhammer, J., \& Hellhammer, D. H. (2005). A new view on hypocortisolism. Psychoneuroendocrinology, 30(10), 1010-1016.

Hatzenbuehler, M. L. (2009). How does sexual minority stigma "get under the skin"? A psychological mediation framework. Psychological Bulletin, 135(5), 707-730.

Hatzenbuehler, M. L., \& McLaughlin, K. A. (2014). Structural stigma and hypothalamic-Pituitary-adrenocortical axis reactivity in lesbian, gay, and bisexual young adults. Annals of Behavioral Medicine, 47(1), 39-47.

Hatzenbuehler, M. L., Slopen, N., \& McLaughlin, K. A. (2014). Stressful life events, sexual orientation, and cardiometabolic risk among young adults in the United States. Health Psychology, 33(10), 1185-1194.

Hayes, A. F. (2008). Introduction to mediation, moderation, and conditional process analysis: A regression-based approach. New York, NY: Guilford Press.

Hazan, C., \& Shaver, P. R. (1994). Attachment as an organizational framework for research on close relationships. Psychological Inquiry, 5, 1-22.

Herrick, A. L., Stall, R., Goldhammer, H., Egan, J. E., \& Mayer, K. H. (2014). Resilience as a research framework and as a cornerstone of prevention research for gay and bisexual men: Theory and evidence. Aids and Behavior, 18(1), 1-9. 
Hunter, A. L., Minnis, H., \& Wilson, P. A. (2011). Altered stress responses in children exposed to early adversity: A systematic review of salivary cortisol studies. Stress, 14, 614-626.

IOM. (2011). The health of lesbian, gay, bisexual, and transgender people: Building a foundation for better understanding. Washinton, DC: The National Academies Press.

Irons, C., Gilbert, P., Baldwin, M. W., Baccus, J. R., \& Palmer, M. (2006). Parental recall, attachment relating and selfattacking/self-reassurance: Their relationship with depression. British Journal of Clinical Psychology, 45, 297-308.

Janssens, K. A., Oldehinkel, A. J., \& Rosmalen, J. G. (2009). Parental overprotection predicts the development of functional somatic symptoms in young adolescents. The Journal of Pediatrics, 154, 918-923.

Jaremka, L. M., Glaser, R., Loving, T. J., Malarkey, W. B., Stowell, J. R., \& Kiecolt-Glaser, J. K. (2013). Attachment anxiety is linked to alterations in cortisol production and cellular immunity. Psychological Science, 24, 272-279.

Juster, R. P., Seeman, T., McEwen, B. S., Picard, M., Mahar, I., Mechawar, N., ... Lupien, S. J. (2016). Social inequalities and the road to allostatic load: From vulnerability to resilience. In D. Cicchetti (Ed.), Developmental psychopathology handbook (3rd ed.). (pp. 381-434). New York, NY: Cambridge Press.

Juster, R.-P., Almeida, D., Cardoso, C., Raymond, C., Johnson, P. J., Pfaus, J. G., ... Lupien, S. J. (2016). Gonads and strife: Sex hormones vary according to sexual orientation for women and stress indices for both sexes. Psychoneuroendocrinology, 72, 119-130.

Juster, R.-P., Bizik, G., Picard, M., Arsenault-Lapierre, G., Sindi, S., Trepanier, L., ... Lupien, S. J. (2011). A transdisciplinary perspective of chronic stress in relation to psychopathology throughout life span development. Development and Psychopathology, 23(3), 725-776.

Juster, R.-P., Hatzenbuehler, M. L., Mendrek, A., Pfaus, J. G., Smith, N. G., Johnson, P. J., ... Pruessner, J. C. (2015). Sexual orientation modulates endocrine stress reactivity. Biological Psychiatry, 77(7), 668-676.

Juster, R.-P., McEwen, B. S., \& Lupien, S. J. (2010). Allostatic load biomarkers of chronic stress and impact on health and cognition. Neuroscience \& Biobehavioral Reviews, 35(1), 2-16.

Juster, R.-P., Ouellet, É., Lefebvre-Louis, J.-P., Sindi, S., Johnson, P. J., Smith, N. G., \& Lupien, S. J. (2016). Retrospective coping strategies during sexual identity formation and current biopsychosocial stress. Anxiety, Stress, \& Coping, 29, $119-138$.

Juster, R.-P., Sindi, S., Marin, M.-F., Perna, A., Hashemi, A., Pruessner, J. C., \& Lupien, S. J. (2011). A clinical allostatic load index is associated with burnout symptoms and hypocortisolemic profiles in healthy workers. Psychoneuroendocrinology, 36(6), 797-805.

Juster, R.-P., Smith, N. G., Ouellet, É., Sindi, S., \& Lupien, S. J. (2013). Sexual orientation and disclosure in relation to psychiatric symptoms, diurnal cortisol, and allostatic load. Psychosomatic Medicine, 75(2), 103-116.

Kajantie, E., \& Phillips, D. I. W. (2006). The effects of sex and hormonal status on the physiological response to acute psychosocial stress. Psychoneuroendocrinology, 31(2), 151-178.

Katz-Wise, S. L., \& Hyde, J. S. (2012). Victimization experiences of lesbian, gay, and bisexual individuals: A metaanalysis. Journal of Sex Research, 49, 142-167.

Kirschbaum, C., Klauer, T., Filipp, S.-H., \& Hellhammer, D. H. (1995). Sex-specific effects of social support on cortisol and subjective responses to acute psychological stress. Psychosomatic Medicine, 57, 23-31.

Kirschbaum, C., Kudielka, B. M., Gaab, J., Schommer, N. C., \& Hellhammer, D. H. (1999). Impact of gender, menstrual cycle phase, and oral contraceptives on the activity of the hypothalamus-pituitary-adrenal axis. Psychosomatic Medicine, 61, 154-162.

Kirschbaum, C., Pirke, K.-M., \& Hellhammer, D. H. (1993). The 'Trier Social Stress Test'-A tool for investigating psychobiological stress responses in a laboratory setting. Neuropsychobiology, 28(1-2), 76-81.

Kirschbaum, C., Wüst, S., \& Hellhammer, D. H. (1992). Consistent sex differences in cortisol responses to psychological stress. Psychosomatic Medicine, 54(6), 648-657.

Klein, F., Sepekoff, B., \& Wolf, T. J. (1990). Sexual orientation: A multivariable dynamic process. In T. Geller (Ed.), Bisexuality: A reader and sourcebook. Ojai, CA: Times Change Press.

Lick, D. J., Durso, L. E., \& Johnson, K. L. (2013). Minority stress and physical health among sexual minorities. Perspectives on Psychological Science, 8, 521-548.

Lima, A. R., Mello, M. F., \& De Jesus Mari, J. (2010). The role of early parental bonding in the development of psychiatric symptoms in adulthood. Current Opinion in Psychiatry, 23(4), 383-387.

Marin, M.-F., Morin-Major, J.-K., Schramek, T. E., Beaupré, A., Perna, A., Juster, R.-P., ... Uddin, M. (2012). There is no news like bad news: Women are more remembering and stress reactive after reading real negative news than men. PLoS One, 7(10), e47189.

McEwen, B. S., \& Stellar, E. (1993). Stress and the individual. Mechanisms Leading to Disease. Archives of Internal Medicine, 153(18), 2093-2101.

McEwen, B. S., \& Wingfield, J. C. (2003). The concept of allostasis in biology and biomedicine. Hormones and Behavior, 43, 2-15.

Meyer, I. H. (2003). Prejudice, social stress, and mental health in lesbian, gay, and bisexual populations: Conceptual issues and research evidence. Psychological Bulletin, 129, 674-697.

Meyer, I. H. (2016). Does an improved social environment for sexual and gender minorities have implications for a new minority stress research agenda? Psychol Sex Rev, 7(1), 81-90. 
Mikulincer, M., \& Shaver, P. R. (2007). Adult attachment: Structure, dynamics, and change. New York: Guilford.

Pachankis, J. E., Goldfried, M. R., \& Ramrattan, M. E. (2008). Extension of the rejection sensitivity construct to the interpersonal functioning of gay men. Journal of Consulting and Clinical Psychology, 76, 306-317.

Parker, G. (1983). Parental overprotection: A risk factor in psychosocial development. New York: Grune \& Stratton.

Parker, G. (1988). The parental bonding instrument: Psychometric properties reviewed. Psychiatric Developments, 7(4), 317-335.

Pietromonaco, P. R., DeBuse, C. J., \& Powers, S. I. (2013). Does attachment get under the skin? Adult romantic attachment and cortisol responses to stress. Current Directions in Psychological Science, 22, 63-68.

Pruessner, J. C., Champagne, F., Meaney, M. J., \& Dagher, A. (2004). Dopamine release in response to a psychological stress in humans and its relationship to early life maternal care: A positron emission tomography study using [11C] raclopride. The Journal of Neuroscience, 24, 2825-2831.

Pruessner, J. C., Kirschbaum, C., Meinlschmid, G., \& Hellhammer, D. H. (2003). Two formulas for computation of the area under the curve represent measures of total hormone concentration versus time-dependent change. Psychoneuroendocrinology, 28(7), 916-931.

Pruessner, M., Vracotas, N., Joober, R., Pruessner, J. C., \& Malla, A. K. (2013). Blunted cortisol awakening response in men with first episode psychosis: Relationship to parental bonding. Psychoneuroendocrinology, 38, $229-240$.

Rosario, M., Reisner, S. L., Corliss, H. L., Wypij, D., Calzo, J., \& Austin, S. B. (2014). Sexual-orientation disparities in substance use in emerging adults: A function of stress and attachment paradigms. Psychology of Addictive Behaviors, 28, 790-804.

Rosario, M., Reisner, S. L., Corliss, H. L., Wypij, D., Frazier, A. L., \& Austin, S. B. (2014). Disparities in depressive distress by sexual orientation in emerging adults: The roles of attachment and stress paradigms. Archives of Sexual Behavior, 43, 901-916.

Rothman, E. F., Sullivan, M., Keyes, S., \& Boehmer, U. (2012). Parents' supportive reactions to sexual orientation disclosure associated with better health: Results from a population-based survey of LGB adults in Massachusetts. Journal of Homosexuality, 59, 186-200.

Ryan, C., Russell, S. T., Huebner, D. M., Diaz, R. M., \& Sanchez, J. (2010). Family acceptance in adolescence and the health of LGBT young adults. Journal of Child and Adolescent Psychiatric Nursing, 23, 205-213.

Saewyc, E. M. (2011). Research on adolescent sexual orientation: Development, health disparities, stigma, and resilience. Journal of Research on Adolescence, 21, 256-272.

Saewyc, E. M., Skay, C. L., Pettingell, S. L., \& Reis, E. A. (2006). Hazards of stigma: The sexual and physical abuse of gay, lesbian, and bisexual adolescents in the United States and Canada. Child Welfare, 85, 195-213.

Sapolsky, R. M. (2002). Endocrinology of the stress-response. In J. B. Becker, S. M. Breedlove, D. Crews, \& M. M. McCarthy (Eds.), Behavioral Endocrinology (2nd ed., pp. 409-450). Cambridge, MA: MIT Press.

Seeman, E., Singer, B. H., Rowe, J., Horwitz, R. I., \& McEwen, B. (1997). Price of adaptation - allostatic load and its health consequences. Archives of Internal Medicine, 157, 2259-2268.

Taylor, S. E., Klein, L. C., Lewis, B. P., Gruenewald, T. L., Gurung, R. A., \& Updegraff, J. A. (2000). Biobehavioral responses to stress in females: Tend-and-befriend, not fight-or-flight. Psychological Review, 107(3), 411-429.

Tsigos, C., \& Chrousos, G. P. (2002). Hypothalamic-Pituitary-Adrenal axis, neuroendocrine factors and stress. Journal of Psychosomatic Research, 53(4), 865-871.

Wadiwalla, M., Andrews, J., Lai, B., Buss, C., Lupien, S. J., \& Pruessner, J. C. (2010). Effects of manipulating the amount of social-evaluative threat on the cortisol stress response in young healthy women. Stress, 13(3), 214-220.

Yanovski, J. A., Yanovski, S. Z., Harrington, L., Gold, P. W., \& Chrousos, G. P. (1995). Differences in the hypothalamicpituitary-adrenal axis of black and white men. Hormone Research in Paediatrics, 44(5), 208-212. 